\title{
In reply: Concomitant cranio-spinal trauma: additional risk from a cerebrovascular injury
}

\author{
Juho Vehviläinen ${ }^{1} \cdot$ Tuomas Brinck $^{2} \cdot$ Matias Lindfors $^{1}$ - Jussi Numminen ${ }^{3} \cdot$ Jari Siironen $^{1} \cdot$ Rahul Raj $^{1}$ (D)
}

Received: 31 October 2020 / Accepted: 3 November 2020 / Published online: 11 November 2020

(C) Springer-Verlag GmbH Austria, part of Springer Nature 2020

Dear Editor,

We thank Demetriades and colleagues for their comments [1], regarding our recently published paper [3]. Briefly, in our paper, we assessed the occurrence of concomitant severe traumatic brain injury (TBI), cervical spine injury (CSI), and/or blunt cerebrovascular injury (BCVI). We also assessed the association between TBI, CSI, BCVI, and 6-month functional outcome and mortality. The presence of concomitant TBI and BCVI was associated with a 6 times increase in odds for unfavorable outcome and an almost eightfold increase in odds for mortality. We did not find any association between CSI and outcome. Below our comments to Demetriades and colleagues:

1. Five TBI patients had a concomitant BCVI and CSI. Thus, of the 16 patients with BCVI, 11 patients (69\%) did not suffer from CSI.

2. Conversely, out of the 26 patients with CSI, only five patients $(19 \%)$ had a BCVI.

3. Due to the small number of patients with TBI, BCVI, and CSI (five patients), one should be careful when interpreting these results. However, all five patients with TBI, BCVI, and CSI had an unfavorable outcome

This article is part of the Topical Collection on Brain trauma

Juho Vehviläinen

juho.vehvilainen@helsinki.fi

$\triangle$ Rahul Raj

rahul.raj@hus.fi

1 Department of Neurosurgery, Helsinki University Hospital and University of Helsinki, Helsinki, Finland

2 Department of Department of Orthopaedics and Traumatology, Helsinki University Hospital and University of Helsinki, Helsinki, Finland

3 Department of Radiology, Helsinki University Hospital and University of Helsinki, Helsinki, Finland
(Glasgow Outcome Score, GOS 1-3) at 6 months, and four patients $(80 \%)$ died.

4. The number of patients with cervical spinal cord injury was too low for any meaningful comparison.

5. Regarding, the local imaging protocol, since 2015, the local imaging protocol has included CT angiography of the cervical spine. We agree that the benefits and possible harms coming from routine "radiological screenings" should be assessed carefully. According to our local imaging protocol, all patients where the trauma alert is triggered (in case of high-energy trauma, physiological derangement, and/or detected anatomical injuries suggesting severe trauma) undergo routine whole-body CT with the so-called split-bolus technique. First, a non-contrast head CT is taken. Second, after a first contrast bolus, the cervical spine and vessels are imaged. Third, after a second contrast bolus, the torso is imaged under combined arterial and venous phase. In other than the trauma alert patients, we are applying the expanded Denver criteria for cervical vessel imaging [2].

\section{References}

1. Demetriades AK, Linnerud H, Gerdhem P, Peul WC (2020) Concomitant cranio-spinal trauma: additional risk from a cerebrovascular injury. Acta Neurochir. https://doi.org/10.1007/s00701020-04573-6

2. Geddes AE, Burlew CC, Wagenaar AE, Biffl WL, Johnson JL, Pieracci FM, Campion EM, Moore EE (2016) Expanded screening criteria for blunt cerebrovascular injury: a bigger impact than anticipated. Am J Surg 212(6):1167-1174

3. Vehviläinen J, Brinck T, Lindfors M, Numminen J, Siironen J, Raj R (2020) Occurrence and prognostic effect of cervical spine injuries and cervical artery injuries with concomitant severe head injury. Acta Neurochir 162(6):1445-1453

Publisher's note Springer Nature remains neutral with regard to jurisdictional claims in published maps and institutional affiliations. 